\title{
Aspectos ecológicos de la regeneración por semillas en bosques nativos de Nothofagus antarctica en Patagonia Sur, Argentina
}

\author{
Ecological aspects of regeneration from seeds of Nothofagus antarctica native forest in Southern \\ Patagonia, Argentina
}

\author{
Héctor A Bahamonde a,b*, Pablo L Peri a,b,c, Lucas H Monelos ${ }^{\text {b }}$, Guillermo Martínez Pastur ${ }^{\mathrm{c}, \mathrm{d}}$ \\ *Autor de correspondencia: ${ }^{a}$ Instituto Nacional de Tecnología Agropecuaria (INTA), cc 332, (CP 9400) Río Gallegos, \\ Santa Cruz, Argentina, tel./fax: 54-2966-442014, habahamonde@correo.inta.gov.ar \\ ${ }^{\mathrm{b}}$ Universidad Nacional de la Patagonia Austral (UNPA), Argentina. \\ ${ }^{c}$ Consejo Nacional de Investigaciones Científicas y Técnicas (CONICET), Argentina. \\ ${ }^{\mathrm{d}}$ Centro Austral de Investigaciones Científicas (CADIC), Argentina.
}

\begin{abstract}
SUMMARY
The aims of the present study were to quantify the annual seed production and its viability in pure stands of Nothofagus antarctica (ñire) grown in three sites, and to evaluate the establishment and mortality of seedling after seeds fall in Southern Patagonia (Argentina). Seed production was sampled using 4 traps in each site during five consecutive years (2004-2008). From this, seed viability and germination were evaluated. In each site, permanent sampling plots were set up for seedling establishment and mortality measurements. Seed production varied from 2 to 52 millions seeds ha ${ }^{-1}$ being higher in better site quality stands. The number of days with air temperature below $0{ }^{\circ} \mathrm{C}$ during the period before flowering explained $42 \%$ of the seed production variation from the sites and years evaluated. Germination varied between sites, being less than $12 \%$ and independent from seed production. Seedlings establishment only occurred in two years with a maximum value of 185,000 seedlings ha-1 but with high mortality at the end of the growing season. We concluded that regeneration from seeds in the years and sites evaluated was not successful. Therefore, a longer period of evaluation is needed to assess the regeneration process in these forests.
\end{abstract}

Key words: seeding, seedling installation, ñire, between years variation.

\section{RESUMEN}

Los objetivos de este estudio fueron cuantificar la producción anual de semillas y su viabilidad germinativa en rodales puros de Nothofagus antarctica (ñire) desarrollándose en tres calidades de sitio, y evaluar el establecimiento y mortalidad de plántulas después de la semillación en Patagonia Sur (51 $\mathrm{S}$, Argentina). La producción de semillas se evaluó con cuatro trampas de captura en cada sitio durante cinco años (2004-2008), determinándose viabilidad y germinación. En cada sitio, se instalaron parcelas permanentes de muestreo para cuantificar la incorporación y supervivencia de plántulas. La producción de semillas por hectárea varió desde dos hasta 52 millones con una tendencia a producir más semillas en los sitios de mejor calidad. El número de días con temperaturas del aire por debajo de $0{ }^{\circ} \mathrm{C}$ en el periodo previo a la floración explicó el $42 \%$ de la variación en la producción de semillas en los sitios y años evaluados. La germinación varió entre sitios siendo independiente de la producción de semillas, no superando el $12 \%$ de promedio en todos los años evaluados. Sólo hubo incorporación de plántulas en dos de los años evaluados con un máximo de 185.000 plántulas por hectárea siendo nula la supervivencia de las plántulas al final de la estación de crecimiento en la mayoría de los casos. Se concluyó que, en los sitios y años evaluados, la regeneración a través de semillas no fue exitosa y se considera necesario continuar evaluando distintos aspectos ecológicos ligados al proceso de regeneración en periodos más largos de tiempo.

Palabras clave: semillación, instalación de plántulas, ñire, variación interanual.

\section{INTRODUCCIÓN}

Nothofagus antarctica (G. Forster) Oerst. (ñire) se extiende latitudinalmente desde los $36^{\circ} 30^{\prime}$ hasta los $56^{\circ}$ $00^{\prime} \mathrm{S}$ y altitudinalmente desde el nivel del mar hasta los 2.000 m s.n.m. (Veblen et al. 1996). Es la especie que alcanza la mayor amplitud ecológica de los Nothofagus spp. sudamericanos (Donoso et al. 2006a), y adopta distintos morfotipos según el ambiente en el que se desarrolla (Ramírez et al. 1985, Premoli 1991). En la Patagonia Sur argentina, existen cerca de 99.000 ha de bosque de $N$. antarctica tipo arbóreo en la provincia de Santa Cruz (Peri 2004) y 181.370 ha en Tierra del Fuego (Collado 2001), distribuidas en un amplio rango de condiciones ambientales. Se han realizado varios estudios en bosques de $N$. antarctica relacionados con aspectos ecológicos y productivos (Gallo et al. 2004, Peri et al. 2005, 2006ab, 2008, Moretto et al. 2006, Navarro Cerrillo 
et al. 2008), pero pocos incluyen aspectos de la ecología de la regeneración de $N$. antarctica en la Patagonia Sur (Soler Esteban et al. 2010), a diferencia de lo que ocurre con la lenga (Nothofagus pumilio (Poep. et Endl) Krasser) (Mascareño 1987, Cuevas 2000, Martínez Pastur et al. 2004, 2008). Esto implica un vacío importante en el conocimiento respecto de la continuidad del estrato arbóreo de $N$. antarctica a través de la regeneración por semillas. La regeneración del bosque es un proceso dinámico que incluye distintas etapas, desde la floración hasta la instalación y supervivencia de plántulas, y cuyo éxito final dependerá de distintos factores bióticos y abióticos que incidirán en cada etapa del proceso (Pulido y Díaz 2005, Martínez Pastur et al. 2008), pudiendo estos factores variar temporal y espacialmente. En Tierra del Fuego, Martínez Pastur et al. (2008) y Soler Esteban et al. (2010) detectaron diferencias en la producción de flores entre distintas estructuras forestales en bosques de $N$. pumilio y $N$. antarctica, respectivamente, lo cual estaría asociado a factores abióticos. También se sabe que en especies de Nothofagus spp. sudamericanos hay una importante pérdida de semillas por caída de frutos inmaduros, depredación predispersión de semillas principalmente por insectos y aves (Donoso 2006) y por depredación posdispersión por mamíferos (Pulido y Díaz 2005). Asimismo hay antecedentes que indican que la instalación y supervivencia de regeneración de $N$. pumilio están influenciadas por la estructura del rodal (Rosenfeld et al. 2006), características del micrositio (Heinemann et al. 2000) o historia de uso (Alauzis et al. 2004), entre otros factores.

Por otro lado, la calidad de un sitio forestal estará definida por la suma de todos los factores ambientales que interactúan y determinan su capacidad productiva (Daniel et al. 1982). Wardle (1984) presenta información acerca de la relación entre calidad de sitio y la producción y calidad de semillas en Nothofagus spp. de Nueva Zelanda. Asimismo, Martínez Pastur et al. (1994) encontraron una relación inversa entre parámetros ligados a la calidad forestal (altura dominante, volumen) y la altitud de bosques de $N$. pumilio en Patagonia argentina, mientras que también existen antecedentes que indican una relación inversa entre la altitud y aspectos ligados a la regeneración de la especie (producción y calidad de semillas, instalación y supervivencia de plántulas) (Cuevas 2000, González et al. 2006).

No obstante, para $N$. antarctica no se conocen antecedentes que relacionen la calidad del sitio forestal con sus aspectos ligados a la regeneración, tales como cantidad y calidad de semillas, instalación y supervivencia de plántulas a través del tiempo. Por ello, en este trabajo se plantea la hipótesis que la producción y viabilidad de las semillas de $N$. antarctica, así como también la instalación y la sobrevivencia de plántulas, estarán influidas por la calidad de sitio y la variación climática interanual. Por lo anterior, los objetivos de este trabajo son: a) cuantificar la producción anual de semillas y su viabilidad germinativa en tres rodales de bosque de ñire desarrollándose en distintas calidades de sitio y b) evaluar el establecimiento y mortalidad de plántulas después de cada periodo de semillación durante cinco años.

\section{MÉTODOS}

Sitios de muestreo. Se seleccionaron tres rodales puros de bosques primarios coetáneos de $N$. antarctica ubicados en la provincia de Santa Cruz creciendo en diferentes calidades de sitio. Para determinar la calidad de sitio de los bosques de $N$. antarctica se utilizó la clasificación propuesta por Lencinas et al. (2002) basada en la altura media de los árboles dominantes, donde las mayores alturas se corresponden con sitios de mejor calidad. Los rodales muestreados fueron: en la estancia Cancha Carrera, uno con clase de sitio II (altura dominante final entre 10 y 12 $\mathrm{m})$, ubicado sobre una ladera de exposición Este (51 ${ }^{\circ} 13^{\text {' }}$ $21^{\prime \prime} \mathrm{S}-72^{\circ} 15^{\prime} 44^{\prime \prime} \mathrm{O}$ ), y otro de clase de sitio IV (altura dominante final entre 6 y $8 \mathrm{~m}$ ), ubicado en una posición de media ladera con exposición Este ( $51^{\circ} 13^{\prime} 22^{\prime \prime} \mathrm{S}-72^{\circ} 15^{\prime}$ 32 " O); y en la estancia Tres Marías un rodal con clase de sitio V (altura dominante final menor a $6 \mathrm{~m}$ ), ubicado en un terreno ondulado lindando con la estepa $\left(51^{\circ} 19^{\prime} 05^{\prime \prime} \mathrm{S}\right.$ - $72^{\circ} 10^{\prime} 47^{\prime \prime} \mathrm{O}$ ). El uso histórico de los rodales estudiados ha sido el de ganadería ovina extensiva desde principios del siglo XX. En la estancia Cancha Carrera el ensayo se instaló en el potrero "Ruca", utilizado en invierno con una carga promedio de 1,6 ovinos hectárea-1 ${ }^{-1}$ correspondientes a ovejas madres. En la estancia Tres Marías el ensayo se ubicó en el potrero "Consumo", siendo éste usado sólo en verano como un sitio de contención de ovinos de manera esporádica (desde 2 a 15 días) de acuerdo a las tareas de manejo que se realizan en la estancia (esquila, señalada, etc.).

El clima corresponde al templado frío húmedo andino, que se caracteriza por temperaturas medias anuales entre 5,5 y $8,0{ }^{\circ} \mathrm{C}$ y precipitaciones (pluviales y nivales) que varían entre 400 y $800 \mathrm{~mm}$ año ${ }^{-1}$ (Soto 2004), con un gradiente ambiental (Oeste - Este) que hace que el rodal de clase de sitio $\mathrm{V}$ presente un régimen hídrico menos favorable, en comparación con los otros dos rodales, con precipitaciones más escasas y mayores velocidades de viento (Bahamonde et al. 2009), lo cual es consistente con su ubicación en el límite con la estepa (figura 1).

Caracterización del estrato arbóreo. En cada sitio de muestreo se realizó una caracterización del estrato arbóreo a partir de tres parcelas circulares de $500 \mathrm{~m}^{2}$ distribuidas al azar, en las cuales se midieron el diámetro normal a 1,3 m (DAP), la altura total (medida con clinómetro y cinta), la cobertura de copas (medida con copímetro de espejo cóncavo; Lemmon 1957) y la cobertura del sotobosque (medida en parcelas de $1 \mathrm{~m}^{2}$ con una red de 25 puntos). A partir de los datos de las parcelas se calculó el área basal y la densidad 


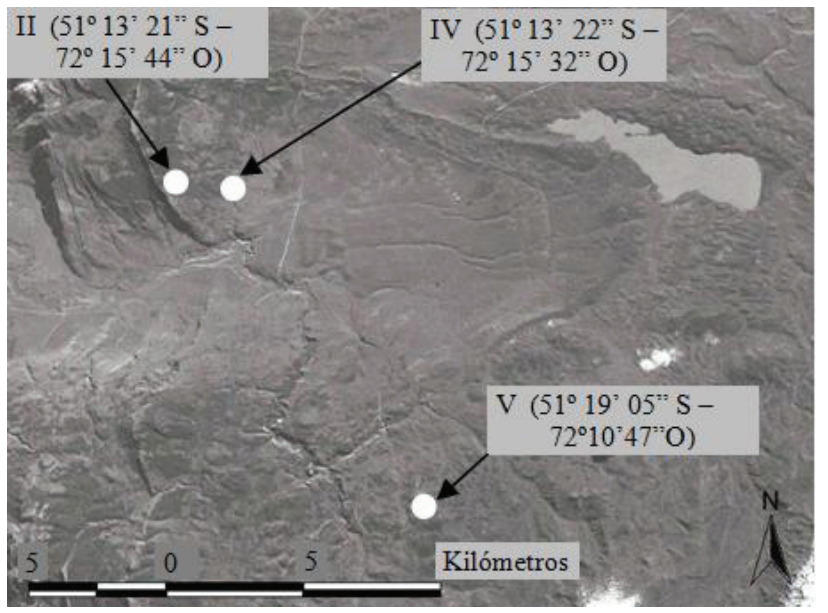

Figura 1. Ubicación de los tres rodales evaluados en distintas clases de sitio: II, IV y V; Santa Cruz, Argentina.

Location of Nothofagus antarctica studied stands grown at different site classes in Santa Cruz province, Argentina: II, IV, V.

de los rodales. Para el cálculo de volumen de los árboles se usaron las ecuaciones propuestas por Lencinas et al. (2002) para distintas clases de sitio de bosques de N. antarctica.

Caracterización del microclima. Las temperaturas del aire $\mathrm{y}$ del suelo, y la humedad relativa del aire se midieron en forma continua cada dos horas (entre junio de 2004 y junio de 2008) en cada rodal. Los sensores de temperatura del aire y humedad relativa se colocaron a una altura de 1 $\mathrm{m}$ del nivel del suelo dentro de una caseta que permite el paso del aire y que además tiene función de protección. La temperatura del suelo se midió mediante termómetros de suelo (HOBO Modelo TMC50-HA, USA) instalados en los primeros $5 \mathrm{~cm}$ del suelo. Con los registros de cada rodal se obtuvieron promedios mensuales. Los datos fueron almacenados mediante data-loggers (HOBO $\mathrm{H} 8$ Family, Onset Computer Corporation, USA).

Producción de semillas. Para cuantificar la producción de semillas se instalaron cuatro trampas de captura de 1 $\mathrm{m}^{2}$ (a $50 \mathrm{~cm}$ de altura sobre el suelo) distribuidas al azar en cada sitio de muestreo; fueron confeccionadas con tela de media sombra y bastidores de madera que han demostrado ser adecuadas para tal fin (Martínez Pastur et al. 2004). Se colectó el material de las trampas en forma mensual durante el período de caída de semillas para estas latitudes (febrero - mayo) entre los años 2004 y 2008. Las muestras se expresaron en número de semillas por unidad de superficie.

Calidad de las semillas. Las semillas fueron pesadas en balanza analítica ( \pm 0,001 g) para determinar el peso de 1.000 semillas. De cada muestra se seleccionaron 40 semillas para clasificarlas de acuerdo a la presencia del endosperma y embrión en llenas y vacías. Posteriormente se efectuó un análisis de viabilidad. Las semillas se embebieron en agua estéril durante 48 horas y se les extrajo el pericarpio, realizando la prueba de viabilidad en las semillas llenas. Los contenidos fueron tratados con trifeniltetrazolium al $1 \%$ durante 48 horas y en oscuridad (Moore 1966). Semillas viables fueron aquéllas que presentaron coloración roja en la zona embrionaria debido a un proceso de reducción que tiene lugar en las células vivas.

Para cuantificar el porcentaje de germinación de las semillas se tomaron al azar de tres a cinco muestras de 100 semillas de cada sitio dependiendo de la disponibilidad de semillas de cada año. En primer lugar se hizo una estratificación de las semillas seleccionadas, manteniéndolas a $4{ }^{\circ} \mathrm{C}$ durante 60 días (Premoli 1991). Posteriormente las semillas estratificadas fueron puestas en una cámara de germinación según las normas ISTA (1999) para Nothofagus spp., durante un periodo de 60 días. El conteo de plántulas (considerándose como tales aquéllas con los dos cotiledones desplegados) se realizó cada dos días.

Regeneración. Para cuantificar la incorporación de plántulas en cada sitio de muestreo, se instalaron cuatro parcelas permanentes de $1 \mathrm{~m}^{2}$ construidas con marcos de hierro (1x1 m) y subdivididas en cuatro cuadrantes (NE, NO, SO, SE) para facilitar la ubicación de cada individuo encontrado en coordenadas X-Y. La mitad de las parcelas permanentes fueron clausuradas con malla de alambre octogonal a fin de aislar el efecto de la ganadería sobre la regeneración. En cada parcela se efectuaron censos al inicio (diciembre) y final (abril) de cada período de crecimiento, midiendo el número de plántulas incorporadas y la tasa de mortalidad de cada periodo. Se consideró el mes de diciembre como inicio de instalación de plántulas debido a que observaciones previas en los rodales estudiados indicaron que no existían plántulas en fechas anteriores.

Análisis estadístico. A fin de evaluar los parámetros estructurales de cada rodal se aplicaron ANOVA y la prueba de Tukey $(P \leq 0,05)$ para separar medias. Para evaluar la producción de semillas y su tamaño (peso de 1.000 semillas) se realizaron ANOVA con medidas repetidas; la clase de sitio fue el factor intersujetos y los años de muestreo el factor intrasujetos (Livacic-Rojas et al. 2006). Para la separación de medias de usó la prueba de Tukey $(P \leq 0,05)$. Con el fin de determinar cuáles podrían ser los factores ambientales de mayor influencia en la variación de la producción de semillas entre años y sitios, se realizaron regresiones lineales simples usando los factores ambientales medidos (temperaturas del aire y del suelo, y humedad relativa del aire) como variables independientes. Para la calidad de semillas (porcentajes de: semillas llenas, viables y germinación) se empleó la prueba no paramétrica de Kruskal-Wallis (por no tener distribución normal), analizando cada año por separado, y la prueba U de Mann-Whitney cuando hubo diferencias significativas entre medias. A fin 
de evaluar si existían asociaciones entre la producción de semillas y los parámetros de calidad evaluados se realizaron análisis de correlación de Spearman. Para todos los análisis estadísticos se usó el software SPSS 11.5.

\section{RESULTADOS}

Estrato arbóreo y microclima. Los parámetros estructurales presentaron cambios de acuerdo a las clases de sitio de los rodales analizados (cuadro 1). La densidad de los rodales fue mayor en los sitios de menor calidad (de II a V). Sin embargo, esta diferencia en la densidad no implicó cambios en la cobertura del dosel superior. La diferencia en las clases de sitio se tradujo en diferencias de las existencias de los rodales expresadas en un rango de volumen total desde $141 \mathrm{~m}^{3} \mathrm{ha}^{-1}(\mathrm{~V})$ a $418 \mathrm{~m}^{3} \mathrm{ha}^{-1}$ (II).

En general los valores medios de temperatura de aire y humedad relativa del aire fueron similares entre sitios, no obstante las temperaturas del aire variaron entre años principalmente en la época invernal (figura 2). En el caso de la temperatura del suelo fue levemente inferior en el rodal de clase de sitio V.

Producción de semillas. La producción de semillas varió entre años $(P=0,034)$, interactuando significativamente con las clases de sitio $(P=0,005)$ (figura $3 \mathrm{~A}$ ). La producción de semillas fue muy variable, con promedios desde dos hasta 52 millones de semillas ha ${ }^{-1}$. Es destacable la alta producción de semillas encontrada en el año 2005 donde los rodales de clases de sitio II y IV produjeron 52 y 43 millones de semillas ha ${ }^{-1}$, respectivamente. El promedio de producción de semillas fue de 10,20 y 21 millones de semillas ha-1 ${ }^{-1}$, para calidades de sitio V, IV y II, respectivamente.

En el análisis del patrón temporal de la caída de semillas no se detectaron diferencias en la distribución mensual entre años y clases de sitio, por lo cual se presentan los valores promedio (figura 3B). La caída de semillas fue máxima en los meses de febrero y marzo en los tres rodales alcanzando un $80 \%$ del total de la caída del periodo estudiado (febrero-mayo).

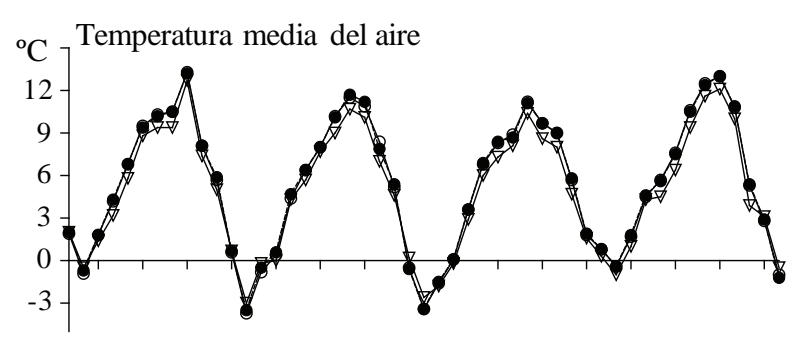

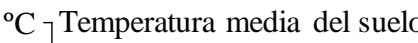
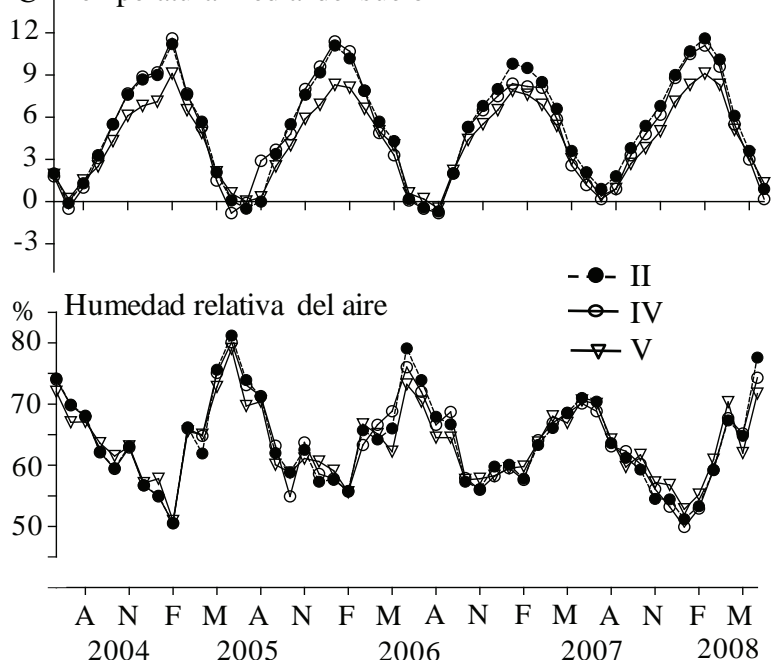

Figura 2. Valores promedios mensuales de temperaturas del aire y del suelo (5 $\mathrm{cm}$ de profundidad), y humedad relativa del aire, entre junio de 2004 y junio de 2008 en los tres rodales de Nothofagus antarctica evaluados. Clases de sitio: II, IV y V. F: febrero, M: mayo, A: agosto, N: noviembre.

Mean monthly air and soil temperatures $(5 \mathrm{~cm}$ depth), and relative air humidity between June 2004 and June 2008 of Nothofagus antarctica stands grown at the site classes studied (II, IV, V). F: February, M: May, A: August, N: November.

Calidad de semillas. El peso de las semillas, que se vincula principalmente con el tamaño de las mismas, no mostró diferencias significativas entre años $(P>0,05)$. En las comparaciones entre sitios en cada año evaluado, los años 2004 y 2008 mostraron que las semillas provenientes del

Cuadro 1. Parámetros estructurales medios de los rodales de Nothofagus antarctica en tres clases de sitio*. Main dasometric parameters of Nothofagus antarctica stands grown at three site classes*.

\begin{tabular}{|c|c|c|c|c|c|c|c|c|c|c|c|}
\hline $\begin{array}{l}\text { Clase de } \\
\text { sitio }\end{array}$ & $\begin{array}{l}\text { HD } \\
\text { (m) }\end{array}$ & $\begin{array}{l}\text { Cobertura } \\
\text { árboles } \\
(\%)\end{array}$ & $\begin{array}{l}\text { Cobertura } \\
\text { sotobos- } \\
\text { que }(\%)\end{array}$ & $\begin{array}{c}\mathrm{N} \\
\left(\text { individuos } \mathrm{ha}^{-1} \text { ) }\right.\end{array}$ & $\mathrm{DAP}(\mathrm{cm})$ & $\begin{array}{c}\mathrm{AB} \\
\left(\mathrm{m}^{2} \mathrm{ha}^{-1}\right)\end{array}$ & $\begin{array}{l}\text { Dom } \\
(\%)\end{array}$ & $\begin{array}{l}\text { Cod } \\
(\%)\end{array}$ & $\begin{array}{l}\text { Int } \\
(\%)\end{array}$ & $\begin{array}{l}\text { Sup } \\
(\%)\end{array}$ & $\begin{array}{c}\text { Vol } \\
\left(\mathrm{m}^{3} \mathrm{ha}^{-1}\right)\end{array}$ \\
\hline II & $11,7 \mathrm{a}$ & $75 a$ & $46 a b$ & $746 b$ & $32,0 \mathrm{a}$ & $63,6 a$ & $26 a b$ & $25 a$ & $26 a$ & $23 b$ & $418 \mathrm{a}$ \\
\hline IV & $7,2 b$ & $85 a$ & $35 b$ & $895 a$ & $24,8 \mathrm{ab}$ & $57,8 \mathrm{ab}$ & $33 a$ & $23 a$ & $27 \mathrm{a}$ & $17 \mathrm{~b}$ & $276 b$ \\
\hline V & $5,0 \mathrm{c}$ & $80 \mathrm{a}$ & $50 a$ & $962 a$ & $19,8 \mathrm{~b}$ & $43,5 b$ & $21 b$ & $28 \mathrm{a}$ & $22 b$ & $29 a$ & $141 c$ \\
\hline
\end{tabular}

*Clases de sitio de acuerdo a Lencinas et al. (2002). HD: altura media de los árboles dominantes; DAP: diámetro normal a 1,3 m; AB: área basal; Dom: árboles dominantes; Cod: árboles codominantes; Int: árboles intermedios; Sup: árboles suprimidos; Vol: volumen. Letras distintas en una misma columna indican diferencias significativas $(P<0,05)$ entre clases de sitio.

*Site classes based on Lencinas et al. (2002). HD: average height dominant trees, Cobertura árboles: crown closure, Cobertura sotobosque: understory cover, N: tree density, DAP: diameter at breast height, AB: basal area, Dom: dominant trees, Cod: co-dominant trees, Int: intermediate trees, Sup: suppressed trees, Vol: volume. Different letters in the same column indicates significant difference among site classes $(P<0.05)$. 
A
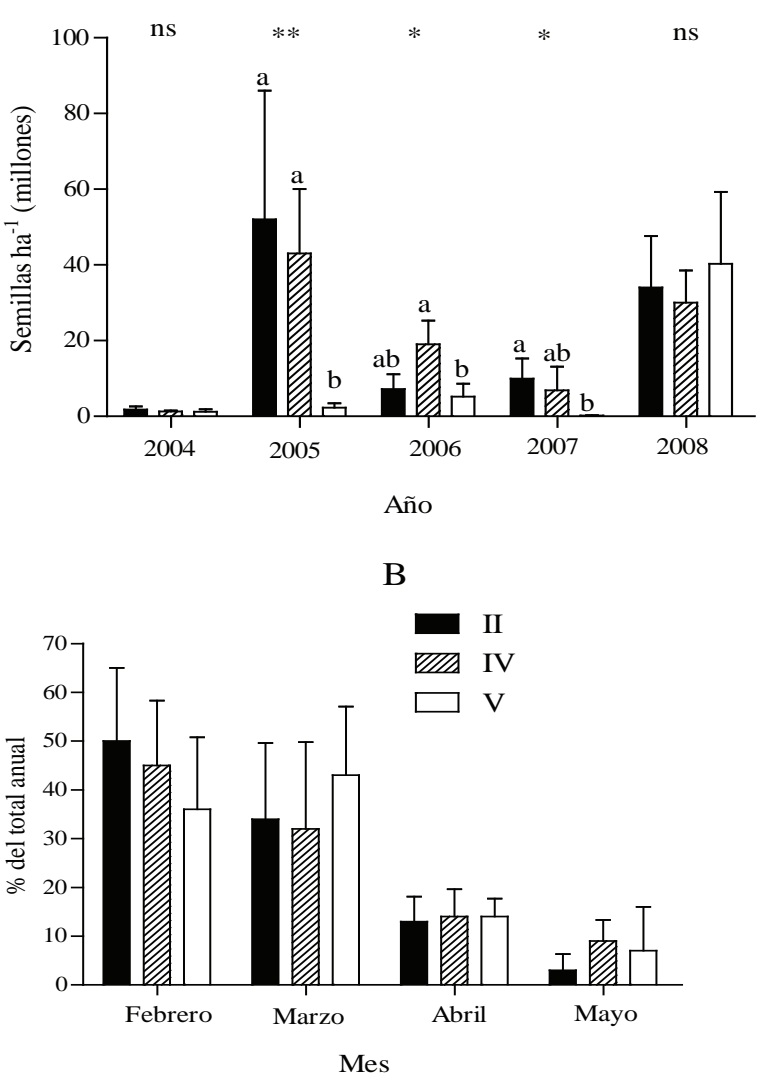

C

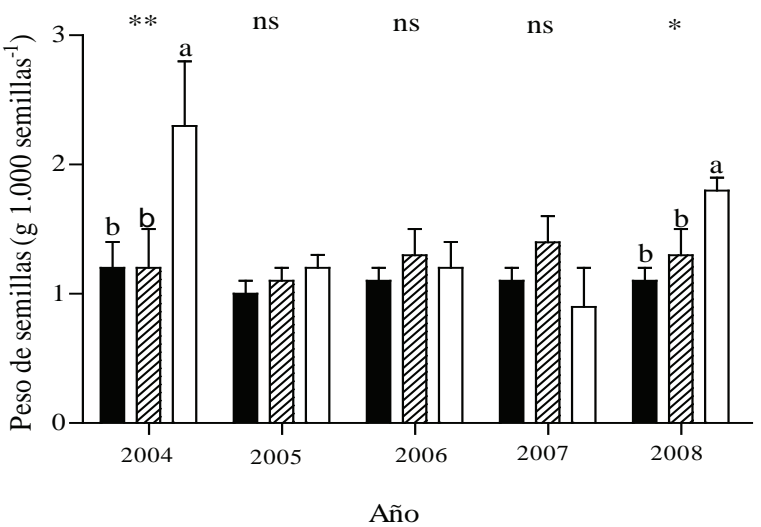

Figura 3. A) Producción anual de semillas (semillas ha-1); B) distribución mensual (promedio del período) de la caída de semillas en porcentaje del total anual y C) peso de semillas (g 1.000 semillas $^{-1}$ ) en bosques de Nothofagus antarctica en tres clases de sitio (V, IV, II) durante cinco años (2004-2008). Las barras representan el desvío estándar de las medias. Letras distintas entre clases de sitio para un mismo año indican diferencias estadísticamente significativas: $* P<0,05,{ }^{*} P<0,01$, ns: no significativo.

A) Annual seed production (seeds ha ${ }^{-1}$ ), B) monthly distribution (mean of period) of seeds fall (expressed as a percentage of total annual seed production) and C) seed weight (g 1,000 seeds ${ }^{-1}$ ) in Nothofagus antarctica stands grown at three site classes during five years (2004-2008). Bars represent standard deviation of the means. Lower case letters indicate significant differences among sites for one particular year: ${ }^{*} P<0.05$, ** $P<0.01$, ns: not significant. rodal de clase de sitio $\mathrm{V}$ fueron las más pesadas que en los rodales de mayor calidad, alcanzando 2,3 y 1,8 g 1.000 semillas $^{-1}$, respectivamente (figura $3 \mathrm{C}$ ).

Tanto los valores de semillas llenas como viables no presentaron diferencias $(P>0,05)$ entre años (la viabilidad del año 2004 no se incluyó debido a falta de semillas) (cuadro 2). Al comparar cada año por separado a través de pruebas no paramétricas tampoco se encontraron diferencias entre clases de sitio debido a su alta variabilidad interna.

Los porcentajes de germinación de semillas provenientes de los rodales variaron dependiendo de las calidades de sitio y del año (cuadro 3). Por ejemplo, en el año 2007 las mejores clases de sitio (II y IV) presentaron mayores valores de germinación, mientras que en los años 2005 y 2008 las semillas colectadas en la clase de sitio $\mathrm{V}$ fueron las de mayor germinación (cuadro 3). El análisis de correlación de Spearman entre diferentes parámetros de calidad y producción de semillas mostró que la germinación de las mismas estaba positivamente correlacionada con su viabilidad $(\mathrm{r}=0,76 ; P<0,01)$. Asimismo se encontraron correlaciones positivas entre la cantidad de semillas producidas y el porcentaje de semillas llenas $(\mathrm{r}=0,57 ; P<0,05)$ y viables $(\mathrm{r}=0,58 ; P<0,05)$.

Del análisis de regresión lineal entre variables ambientales y producción de semillas surgió que el número de días con temperaturas del aire bajo cero entre junio y agosto (previo a la floración) fue el factor ambiental que explicó la mayor parte de la variación en la posterior producción de semillas (figura 4). Es necesario indicar que el modelo obtenido se basa en un rango de entre 34 y 61 días con temperaturas menores a $0{ }^{\circ} \mathrm{C}$ en el periodo de floración.

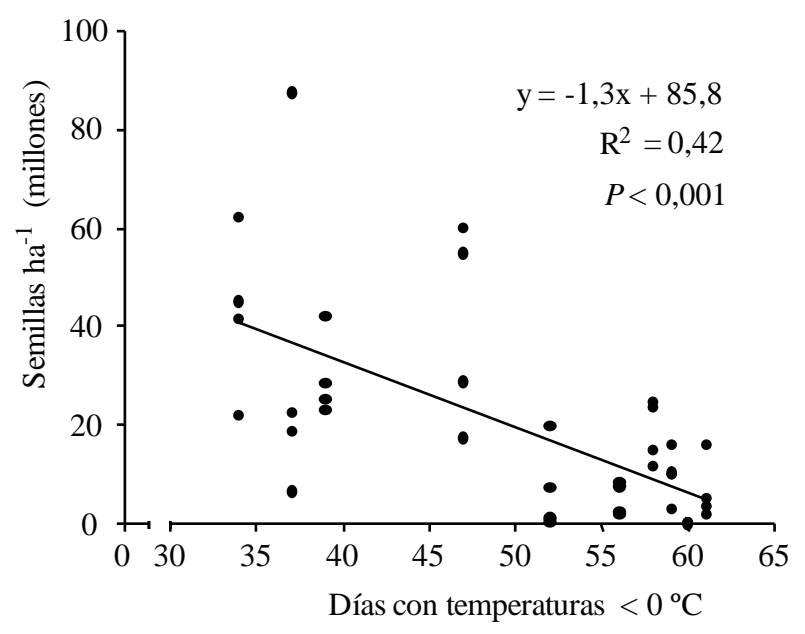

Figura 4. Relación entre la producción de semillas ha-1 de $N$. antarctica y número de días con temperaturas del aire menores a $0{ }^{\circ} \mathrm{C}$ durante el periodo previo a la floración (junio-agosto) del año anterior.

Relationship between seed production (seeds ha ${ }^{-1}$ ) and days with air temperature bellow $0{ }^{\circ} \mathrm{C}$ during previous flowering period (JuneAugust). 
Cuadro 2. Porcentajes (media \pm desviación estándar) $)^{\#}$ de semillas llenas y viables de $N$. antarctica en tres clases de sitio durante 5 y 4 años de medición, respectivamente.

Sound and viable seeds percentages of Nothofagus antarctica (average \pm standard deviation) ${ }^{\#}$ grown at three site classes during 5 and 4 years of measurements, respectively.

\begin{tabular}{lccccccccc}
\hline \multirow{2}{*}{ Clase de sitio Semillas llenas } & \multicolumn{4}{c}{ Semillas viables } \\
\cline { 2 - 10 } & 2004 & 2005 & 2006 & 2007 & 2008 & 2005 & 2006 & 2007 & 2008 \\
\hline II & $21 \pm 9$ & $45 \pm 18$ & $35 \pm 12$ & $61 \pm 21$ & $42 \pm 14$ & $23 \pm 10$ & $10 \pm 3$ & $24 \pm 9$ & $21 \pm 9$ \\
IV & $39 \pm 9$ & $58 \pm 22$ & $15 \pm 8$ & $42 \pm 14$ & $38 \pm 9$ & $25 \pm 10$ & $5 \pm 3$ & $19 \pm 5$ & $14 \pm 6$ \\
V & $33 \pm 15$ & $20 \pm 8$ & $25 \pm 6$ & $27 \pm 10$ & $63 \pm 17$ & $13 \pm 3$ & $5 \pm 3$ & $10 \pm 4$ & $48 \pm 18$ \\
\hline
\end{tabular}

"Sin diferencias significativas entre sitios (prueba no paramétrica de Kruskal- Wallis; $P<0,05$ ).

"Kruskal Wallis analysis $(P<0.05)$; ns: not significant.

Cuadro 3. Porcentajes de germinación (media \pm desviación estándar) de semillas de $N$. antarctica provenientes de tres rodales ubicados en distintas clases de sitio durante cinco años de muestreo.

Germination percentages of seeds of Nothofagus antarctica stands grown at three site classes during five years of measurements.

\begin{tabular}{cccccc}
\hline Clase de sitio & $\begin{array}{c}2004 \\
(\mathrm{n}=5)\end{array}$ & $\begin{array}{c}2005 \\
(\mathrm{n}=4)\end{array}$ & $\begin{array}{c}2006 \\
(\mathrm{n}=4)\end{array}$ & $\begin{array}{c}2007 \\
(\mathrm{n}=3)\end{array}$ & \multicolumn{1}{c}{$\begin{array}{c}2008 \\
(\mathrm{n}=4)\end{array}$} \\
\hline II & $0,4 \pm 0,9$ & $0,5 \pm 1,0 \mathrm{~b}$ & $2,5 \pm 0,6 \mathrm{~b}$ & $7,0 \pm 2,6 \mathrm{a}$ & $5,5 \pm 2,1 \mathrm{~b}$ \\
$\mathrm{IV}$ & $2,0 \pm 1,4$ & $1,5 \pm 1,0 \mathrm{~b}$ & $4,5 \pm 1,1 \mathrm{a}$ & $9,3 \pm 4,0 \mathrm{a}$ & $1,6 \pm 0,5 \mathrm{c}$ \\
$\mathrm{V}$ & $2,8 \pm 1,8$ & $10,5 \pm 4,4 \mathrm{a}$ & $1,9 \pm 0,5 \mathrm{~b}$ & $0,6 \pm 1,0 \mathrm{~b}$ & $43,0 \pm 4,9 \mathrm{a}$ \\
& $\mathrm{ns}$ & $*$ & $*$ & $*$ & $* *$ \\
\hline
\end{tabular}

Letras distintas en una misma columna indican diferencias significativas entre clases de sitio: ${ }^{*} P<0,05, * * P<0,01$, ns: no significativo.

Different letters in the same column indicates significant difference among site classes: $* P<0.05$, ${ }^{* *} P<0.01$, ns: not significant.

Regeneración. Debido a que no se encontraron diferencias en la incorporación de plántulas entre las parcelas con y sin protección para ganado, los datos se presentan como el promedio de las cuatro parcelas indistintamente. En el año 2006 hubo incorporación de plántulas sólo en el rodal de clase de sitio II (10.000 plántulas ha-1), sin supervivencia al final del periodo de crecimiento. En cambio, en el año 2008 hubo regeneración en los rodales de clases de sitio II y V con 5 mil y 180 mil plántulas por hectárea, respectivamente. En el rodal de clase de sitio IV no hubo incorporación de plántulas en ninguno de los años estudiados.

El establecimiento de plántulas fue independiente de la producción de semillas de cada clase de sitio y año, siendo en general muy bajo con un máximo de 180.000 plántulas ha-1 en el rodal de clase de sitio V el año 2008. Esto correspondería sólo a un 0,5\% del total de las semillas producidas y al $1,1 \%$ de la germinación máxima alcanzada para dicho sitio en condiciones de laboratorio. Asimismo, la supervivencia de plántulas al final del periodo de crecimiento fue también muy baja, con valores de 5 mil y 35.000 plántulas ha ${ }^{-1}$ en las clases de sitio II y $\mathrm{V}$, respectivamente.

\section{DISCUSIÓN}

La gran variabilidad en la producción de semillas entre años ha sido documentada también en otros Nothofagus spp. sudamericanos (Marchelli y Gallo 1999, Frangi et al. 2004, Donoso et al. 2006b). En ellos se ha logrado establecer cierta periodicidad cíclica entre años de alta producción de semillas, cuya duración o magnitud dependería de cada especie, las condiciones ambientales del lugar (humedad, temperatura, viento, exposición, etc.), factores bióticos (insectos o enfermedades) y aspectos genéticos (Marchelli y Gallo 1999). Sin embargo, no se conocían hasta el momento datos de este tipo para $N$. antarctica, existiendo solo un antecedente en Tierra del Fuego donde se evaluó la floración y producción de semillas durante un año en bosques de esta especie bajo distinto uso (Soler Esteban et al. 2010). En este sentido, en el presente estudio no fue posible vislumbrar tal periodicidad en ninguno de los rodales estudiados. Se debe tener presente que es muy probable que cinco años de mediciones no sean suficientes para esta especie o que, como concluyeron Herrera et al. (1998), las variaciones en la semillación de especies leñosas no respondan a patrones temporales cíclicos dada la complejidad del proceso. Por otro lado, también se pudo apreciar gran variabilidad en la producción anual de semillas (figura 3) con una tendencia a mayores producciones en los sitios de mejor calidad (II y IV). No obstante, destaca la alta producción de semillas en clase de sitio V en el año 2008, con 40 millones de semillas por hectárea. Este rodal está ubicado en el límite con la estepa patagónica en condiciones más adversas de balance hídrico (mayor evapotranspiración) (Bahamonde et al. 2009).

El comportamiento de gran variabilidad en la 
producción de semillas entre años y sitios también ha sido documentado para otros Nothofagus spp. en un amplio rango de condiciones ambientales (Wardle 1970, Allen 1987, Alley et al. 1998, Marchelli y Gallo 1999).

Los resultados obtenidos sugieren que la distribución temporal de la caída de semillas (figura 3B) es independiente de su producción total para los sitios estudiados. Estos picos de caída de semillas durante algún mes en particular estarían controlados por los tiempos de floración de los árboles (Manson 1974), que en este caso ocurren entre mediados de septiembre y octubre. Este comportamiento de patrón estacional de caída de semillas es similar para $N$. pumilio en Tierra del Fuego (Martínez Pastur et al. 2004) y para otros Nothofagus spp. no sudamericanos (Wardle 1970, Manson 1974).

El rango de valores encontrados en peso de mil semillas (figura 3C) se encuentra dentro de los valores documentados en otros estudios realizados para la especie en latitudes más septentrionales (Donoso y Cabello 1978, Premoli 1991), pero inferiores a N. pumilio y otros Nothofagus spp. (Allen 1987, Beggs 1999, González et al. 2008). Por otro lado, el tamaño de las semillas resulta independiente de la producción de las mismas y no se correlaciona con su posterior germinación, encontrándose diferencias entre sitios sólo en dos de los años evaluados (2004 y 2008), donde las semillas del rodal con calidad de sitio $\mathrm{V}$ son comparativamente más pesadas que las de los otros rodales (figura 3C). Marcheli y Gallo (1999) reportan resultados similares en cuanto a la interacción entre poblaciones y años, al evaluar el tamaño de semillas de Nothofagus nervosa (Phil.) Dim. et Mil. en el norte de Patagonia argentina, y atribuyen dichos resultados a variaciones genéticas e influencias ambientales.

Los porcentajes de semillas llenas y viables encontrados están en el rango de los reportados por Soler Esteban et al. (2010), quienes determinaron valores de viabilidad entre 13 y $21 \%$ en bosques de $N$. antarctica en Tierra del Fuego, y son relativamente altos en comparación con los encontrados por Premoli (1991) para la especie, cuyo valor máximo de semillas viables fue de $13 \%$. Probablemente esta diferencia en viabilidad tenga relación con la alta producción de semillas encontrada en este trabajo y su correlación positiva con la cantidad de semillas llenas y viables. En este sentido, Cuevas (2000) reportó mayor cantidad de semillas llenas y viables en años de mayor producción para bosques de $N$. pumilio creciendo a distintas altitudes en Tierra del Fuego. Estas correlaciones positivas entre producción y calidad de semillas podrían estar favorecidas por factores bióticos como daño por insectos o aves (Martínez Pastur et al. 2008), que podrían verse relativamente minimizados en años de altas producciones de semillas.

Mas allá de la variabilidad en semillas llenas y viables, los valores encontrados de germinación en general no superan el $10 \%$, confirmando datos bibliográficos sobre la baja germinación que presenta $N$. antarctica (Donoso y Cabello 1978, Premoli 1991) comparada con la de otros Nothofagus spp. sudamericanos (Donoso y Cabello 1978, Ordóñez 1986, Acevedo y Urra 2002). Excepcionalmente, el rodal de calidad de sitio $\mathrm{V}$ presenta alta germinación en el año 2008 (cuadro 3), siendo la mayor para la especie de acuerdo a la bibliografía existente, y coincide con su máxima producción de semillas (figura 3A). Como era esperable, la germinación presenta alta correlación positiva con la viabilidad de las semillas.

La relación significativa entre la producción de semillas y el número de días con temperaturas del aire bajo cero entre junio y agosto (previo a la floración) (figura 4) tendría su fundamento fisiológico en que una mayor cantidad de días con temperaturas muy bajas en el periodo de vernalización inhibiría la ulterior floración (Salisbury y Ross 1992) con la consecuente menor producción de semillas. En este sentido Soler Esteban et al. (2010) encontraron menor producción de flores masculinas en bosques de $N$. antarctica bajo uso silvopastoril en comparación con bosques primarios en Tierra del Fuego, lo cual atribuyen a temperaturas más adversas en los rodales bajo uso silvopastoril. No obstante, se considera que esta información del presente trabajo debe ser mantenida sólo como hipótesis para posteriores investigaciones, ya que no se ha medido floración en este estudio.

Los valores de incorporación de plántulas son inferiores a los encontrados por Tejera et al. (2005) en un bosque de $N$. antarctica en la provincia de Chubut (Argentina), quienes encontraron valores de incorporación de 2.000 a 8.500 .000 plántulas $\mathrm{ha}^{-1}$. Teniendo en cuenta que las plántulas de Nothofagus spp. se establecen mejor bajo condiciones moderadamente altas de luz (Veblen et al. 1996), la alta cobertura de copas de los rodales estudiados (cuadro 1) podría ser una de las causas de esta baja incorporación de plántulas. Este efecto del nivel de luz sobre la regeneración de $N$. antarctica ha sido documentado en otros estudios en la Patagonia argentina (Tejera et al. 2005, Peri et al. 2006a) que encontraron mayor número de incorporación de plántulas en bosques con menor cobertura arbórea; se reportan rangos de 0 a 275.000 plántulas ha ${ }^{-1}$ en bosques con coberturas de copas de $80 \%$ y de 350 a 600 mil plántulas por hectárea para los mismos sitios con coberturas de copa de $50 \%$. Sin embargo, existen otros antecedentes para $N$. pumilio que indican que esa especie tiene establecimiento de plántulas independientemente del nivel de luz en el límite xérico de su distribución en el noroeste de la Patagonia argentina (Heinemann et al. 2000).

De manera similar a lo encontrado en este trabajo, Tejera et al. (2005) y Peri et al. (2006a) reportan baja o nula supervivencia de plántulas en bosques de $N$. antarctica bajo distintos usos. En este sentido, varios autores informan que para $N$. pumilio la humedad del suelo sería un factor determinante en la instalación y supervivencia de plántulas (Rusch 1992, Cuevas 2000, Heinemann et al. 2000, Tercero-Bucardo et al. 2007), lo 
cual podría explicar en parte los resultados obtenidos en este trabajo considerando que en los sitios evaluados las precipitaciones no superan los $800 \mathrm{~mm}$ anuales.

Si se considera que, en general, la especie tendría una alta mortalidad de individuos en los primeros 10 años (Donoso et al. 2006a), los valores encontrados en estos rodales no garantizarían su continuidad en el tiempo a través de la regeneración por semillas. Esto confirmaría la alta dependencia que tiene la especie de la regeneración vegetativa (Veblen et al. 1996, Donoso et al. 2006a), independientemente de que su producción de semillas sea alta. En este mismo sentido, existen antecedentes que indican que en la Patagonia argentina $N$. antarctica puede rebrotar desde las raíces después de eventos de incendios en la zona ecotonal con la estepa (Donoso et al. 2006a).

\section{CONCLUSIONES}

La producción de semillas de $N$. antarctica varió entre años mostrando los rodales de clases de sitio más altas (II y IV) una tendencia a producir mayor cantidad de semillas que en el sitio de menor calidad (V). En los cinco años de medición no se aprecia una respuesta cíclica en la producción de semillas como se ha visto en otros Nothofagus spp.

En general, la germinación de semillas de $N$. antarctica es baja comparada con la de otros Nothofagus spp. sudamericanos, independientemente de la cantidad y tamaño de las mismas.

A pesar de la muy baja o nula supervivencia de plántulas encontradas en este trabajo es necesario continuar con este tipo de mediciones para tener una idea más acertada acerca de la potencial continuidad del estrato arbóreo a través de la regeneración por semillas.

\section{AGRADECIMIENTOS}

Se agradece a: Daniel Ledesma, Fernando Coleahue, Alejandro Ojeda, Sabrina Billoni y Javier Szewczuk por su aporte en la recolección y procesamiento de muestras; a Santiago Fernández por permitir la realización de este trabajo en los sitios estudiados.

\section{REFERENCIAS}

Acevedo S, C Urra. 2002. Caracterización de procedencias en la etapa de viverización de Nothofagus alessandri Espinosa (ruíl) y Nothofagus glauca (Phil.) Krasser (hualo). Seminario de Título Ingeniería Forestal. Talca, Chile. Facultad de Ciencias Agrarias y Forestales, Universidad Católica del Maule. $76 \mathrm{p}$.

Alauzis MV, MJ Mazzarino, E Raffaele, L Roselli. 2004. Wildfires in NW Patagonia: long-term effects on a Nothofagus forest soil. Forest Ecology and Management 192: 131-142.

Allen RB. 1987. Ecology of Nothofagus menziesii in the Catlins
Ecological Region, South-east Otago, New Zealand (I) Seed production, viability, and dispersal. New Zealand Journal of Botany 25(11): 5-10.

Alley JC, BM Fitzgerald, PH Berben, SJ Haslett. 1998. Annual and seasonal patterns of litter-fall of hard beech (Nothofagus truncata) and silver beech (Nothofagus menziesii) in relation to reproduction. New Zealand Journal of Botany 36(33): 453-464.

Bahamonde HA, PL Peri, G Martínez Pastur, V Lencinas. 2009. Variaciones microclimáticas en bosques primarios y bajo uso silvopastoril de Nothofagus antarctica en dos Clases de Sitio en Patagonia Sur. In Primer congreso silvopastoril, Posadas, Misiones, Argentina mayo de 2009. Actas. p. 289296.

Beggs JR. 1999. Comparison of the quality of red and silver beech (Nothofagus) seeds in Nelson Lakes Nacional Park, New Zealand. New Zealand Journal of Botany 37(33): 495501.

Collado L. 2001. Los Bosques de Tierra del Fuego. Análisis de su estratificación mediante imágenes satelitales para el inventario forestal de la Provincia. Multequina 10: 1-16.

Cuevas JG. 2000. Tree recruitment at the Nothofagus pumilio alpine timberline in Tierra del Fuego, Chile. Journal of Ecology 88(5): 840-855.

Daniel PW, UE Helms, FS Baker. 1982. Principios de silvicultura. 2da ed. México DF, México. McGraw Hill. 492 p.

Donoso C, A Cabello. 1978. Antecedentes fenológicos y de germinación de especies leñosas chilenas. Ciencias Forestales 1(2): 31-41.

Donoso C. 2006. Floración, fructificación y semillación. In Donoso Zegers $\mathrm{C}$ ed. Las especies arbóreas de los bosques templados de Chile y Argentina. Autoecología. Valdivia, Chile. Marisa Cuneo Ediciones. p. 21-28.

Donoso C, L Steinke, A Premoli. 2006a. Nothofagus antarctica (G. Forster) Oerst. In Donoso Zegers C ed. Las especies arbóreas de los bosques templados de Chile y Argentina. Autoecología. Valdivia, Chile. Marisa Cuneo Ediciones. p. 401-410.

Donoso P, C Donoso, C Navarro, B Escobar. 2006b. Nothofagus dombeyi (Mirb.) Oerst. In Donoso Zegers C ed. Las especies arbóreas de los bosques templados de Chile y Argentina. Autoecología. Valdivia, Chile. Marisa Cuneo Ediciones. p. 423-432.

Frangi JL, MD Barrera, J Puigdefábregas, PF Yapura, AM Arambarri, L Richter. 2004. Ecología de los bosques de Tierra del Fuego. In Arturi M, J Frangi, JF Goya eds. Ecología y Manejo de los Bosques de Argentina. La Plata, Argentina. Editorial de la Universidad Nacional de La Plata. $88 \mathrm{p}$.

Gallo E, MV Lencinas, PL Peri. 2004. Desarrollo de sistemas silvopastoriles en bosques de Nothofagus antarctica. Biodiversidad en ñirantales. Consultado 20 abr. 2009. Disponible en http://www.ambiente.gov.ar/archivos/web/ PBVyAP/File/A3/PIARFON\%20BAP/Biodiversidad\%20 ire.pdf.

González ME, C Donoso Z, P Ovalle, G Martínez-Pastur. 2006. Nothofagus pumilio (Poep. et Endl) Krasser. In Donoso Zegers $\mathrm{C}$ ed. Las especies arbóreas de los bosques templados de Chile y Argentina. Autoecología. Valdivia, Chile. Marisa Cuneo Ediciones. p. 486-500.

Heinemann K, T Kitzberger, T Veblen. 2000. Influences of gap 
microheterogeneity on the regeneration of Nothofagus pumilio in a xeric old-growth forest of northwestern Patagonia, Argentina. Canadian Journal of Forest Research 30(1): 25-31.

Herrera CM, P Jordano, J Guitián, A Traveset. 1998. Annual Variability in Seed Production by Woody Plants and the Masting Concept: Reassessment of Principles and Relationship to Pollination and Seed Dispersal. The American Naturalist 152(4): 576-594.

ISTA (International Rules for Seed Testing). 1999. Internacional Seed Association Rules. Seed Science and Technology 27: 50-52.

Lemmon P. 1957. A new instrument for measuring forest overstory density. Journal of Forestry 55: 667-668.

Lencinas MV, G Martínez Pastur, JM Cellini, R Vukasovic, PL Peri, MC Fernández. 2002. Incorporación de la altura dominante y clase de sitio a ecuaciones estándar de volumen para Nothofagus antarctica. (Forster f.) Oersted. Bosque 23(2): 5-17.

Livacic-Rojas P, G Vallejo, P Fernández. 2006. Procedimientos estadísticos alternativos para evaluar la robustez mediante diseños de medidas repetidas. Revista Latinoamericana de Psicología 38: 579-598.

Manson BR. 1974. The life history of silver beech (Nothofagus menziesii). Proceedings of the New Zealand Ecological Society 21: 27-31.

Marchelli P, LA Gallo. 1999. Annual and geographic variation in seed traits of Argentinean Populations of southern beech Nothofagus nervosa (Phil.) Dim. et Mil. Forest ecology and Management 121(3): 239-250.

Martínez Pastur G, MC Fernández, PL Peri. 1994. Variación de Parámetros estructurales y de composición del sotobosque para bosques de Nothofagus pumilio en relación a gradientes ambientales indirectos. Ciencias Forestales 9: 11-22.

Martínez Pastur G, A Saenz, SM Rivera, JM Cellini, A Moretto, MV Lencinas, E Gallo, M Villareal. 2004. Aspectos relacionados con la regeneración del bosque intervenido mediante distintos tipos de corta. Proyecto. Alternativas de Manejo Sustentable para el Manejo Forestal Integral de los bosques de Patagonia. Consultado 16 feb. 2009. Disponible en http://www.ambiente.gov.ar/archivos/web/PBVyAP/ File/A3/PIARFON\%20BAP/Regeneracion\%20lenga.pdf.

Martínez Pastur G, MV Lencinas, PL Peri, JM Cellini. 2008. Flowering and seeding patterns in unmanaged and manager Nothofagus pumilio south Patagonian forests. Forstarchiv 79: 60-65.

Mascareño A. 1987. Evaluación de ensayos de semillación y regeneración de Lenga (Nothofagus pumilio Poepp. et Endl. Krasser) bajo diferentes tratamientos a la cama de semillas en la reserva forestal Trapananda, Coyhaique, XI Región. Tesis Ingeniero Forestal. Valdivia, Chile. Facultad de Ciencias Forestales, Universidad Austral de Chile. 95 p.

Moore RP, 1966. Tetrazolium tests for diagnosing causes for seed weaknesses and for predicting and understanding performance. In Proceedings of the Association of Official Seed Analysts of North America 56. p. 70.

Moretto A, J Escobar, PL Peri, R Taier. 2006. Producción, descomposición y mineralización en bosques de Nothofagus antarctica con uso silvopastoril en Tierra del Fuego. In Actas XXII Reunión Argentina de Ecología (ASAE). Córdoba, Argentina, agosto de 2006. p. 270.
Navarro Cerrillo RM, M Rosenfeld, J Pérez Aranda, E Padrón, JR Guzmán, R Hernández Clemente, L González. 2008. Evaluación de la mortalidad de bosques de ñirre (Nothofagus antarctica) en la Patagonia chilena mediante imágenes Landsat TM y ETM+. Bosque 29(1): 65-73.

Ordóñez A. 1986. Germinación de las tres especies de Nothofagus siempreverdes (Coigües) y variabilidad en la germinación de procedencias de Coigüe común (Nothofagus dombeyi (Mirb.) Oerst.). Tesis Ingeniero Forestal. Valdivia, Chile. Facultad de Ciencias Forestales, Universidad Austral de Chile. 144 p.

Peri PL. 2004. Bosque nativo. In González L, Rial P eds. Guía geográfica interactiva de Santa Cruz. Buenos Aires, Argentina. Editorial Instituto Nacional de Tecnología Agropecuaria. p. 43-47.

Peri PL, MV Sturzenbaum, L Monelos, E Livraghi, R Christiansen, A Moreto, JP Mayo. 2005. Productividad de sistemas silvopastoriles en bosques nativos de ñire (Nothofagus antarctica) de Patagonia Austral. In Actas III Congreso Forestal Argentino y Latinoamericano. Corrientes, Argentina septiembre de 2005. CD-ROM.

Peri PL, LH Monelos, HA Bahamonde. 2006a. Evaluación de la continuidad del estrato arbóreo en bosques nativos de Nothofagus antarctica bajo uso silvopastoril con ganado ovino en Patagonia Sur, Argentina. In Actas IV Congreso Latinoamericano de Agroforestería para la Producción Pecuaria Sostenible. Varadero, Cuba, octubre de 2006. p. 87-92.

Peri PL, V Gargaglione, G Martínez Pastur. 2006b. Dynamics of above- and below-ground biomass and nutrient accumulation in an age sequence of Nothofagus antarctica forest of Southern Patagonia. Forest Ecology and Management 233: 85-99.

Peri PL, HA Bahamonde, LH Monelos, G Martínez Pastur. 2008. Producción de Hojarasca en Bosques Primarios y bajo Manejo Silvopastoril de Nothofagus antarctica en la Provincia de Santa Cruz, Argentina. In Segunda reunión sobre Nothofagus en la Patagonia, EcoNothofagus 2008. Esquel, Argentina. p. 149-155.

Premoli A. 1991. Morfología y capacidad germinativa en poblaciones de Nothofagus antarctica (Forster) Oerst del noroeste andino patagónico. Bosque 12(2): 53-59.

Pulido F, M Díaz. 2005. Regeneration of Mediterranean oak: A whole-cycle approach. Ecoscience 12: 92-102.

Ramírez C, M Correa, H Figueroa, J San Martín. 1985. Variación del hábito y hábitat de Nothofagus antarctica en el sur de Chile. Bosque 6(2): 55-73.

Rosenfeld JM, RM Navarro Cerrillo, JR Guzman Alvarez. 2006. Regeneration of Nothofagus pumilio (Poep. et Endl) Krasser forest after five years of seed tree cutting. Journal of Environmental Management 78 : 44-51.

Rusch V. 1992. Principales limitantes para la regeneración de la lenga en la zona N.E. de su área de distribución. Variables ambientales en claros del bosque. In Actas del Seminario de Manejo Forestal de la Lenga y Aspectos Ecológicos Relacionados. Esquel. Argentina. Vol. N 8. p. 61-73.

Salisbury FB, CW Ross. 1992. Plant Physiology. California, USA. Wadsworth Publishing Company. 682p.

Soler Esteban R, G Martínez Pastur, MV Lencinas, PL Peri. 2010. Flowering and seeding patterns in primary, secondary and silvopastoral managed Nothofagus antarctica forests in 
South Patagonia. New Zealand Journal of Botany 48(2): 63-73.

Soto J. 2004. Clima. In González L y Rial P eds. Guía geográfica interactiva de Santa Cruz. Buenos Aires, Argentina. Editorial Instituto Nacional de Tecnología Agropecuaria. p. 25-28.

Tejera L, N Hansen, M Fertig. 2005. Efecto de la cobertura arbórea y del pastoreo vacuno sobre el establecimiento de la regeneración de Nothofagus antarctica (G. Forst.) Oerst. In Actas III Congreso Forestal Argentino y Latinoamericano. Corrientes, Argentina, septiembre de 2005. CD-ROM.

Tercero-Bucardo N, T Kitzberger, TT Veblen, E Raffaele. 2007. A field experiment on climatic and herbivore impacts on post-fire tree regeneration in north-western Patagonia. Journal of Ecology 95:771-779.

Veblen TT, C Donoso, T Kitzberger, AJ Rebertrus. 1996. Ecology of Southern Chilean and Argentinean Nothofagus forests. In Veblen TT, RS Hill, J Read eds. The ecology and biogeography of Nothofagus forests. London, England. Yale University Press. p. 293-353.

Wardle J. 1970. The Ecology of Nothofagus solandri. New Zealand Journal of Botany 8: 571-608.

Wardle J. 1984. The New Zealand Beeches: ecology, utilization and management. Wellington, New Zealand. New Zealand Forest Service. $437 \mathrm{p}$.

Recibido: 11.06 .10

Aceptado: 28.09.10 